\title{
Observations and simulations of asymmetric magnetic field reconnection
}

\author{
F. S. Mozer, ${ }^{1}$ P. L. Pritchett, ${ }^{2}$ J. Bonnell, $^{1}$ D. Sundkvist, ${ }^{1}$ and M. T. Chang ${ }^{1}$ \\ Received 24 June 2008; revised 25 August 2008; accepted 3 September 2008; published 29 November 2008.
}

[1] Comparisons of asymmetric magnetic field reconnection at the subsolar magnetopause are made between satellite data and computer simulations for the ratio of the antiparallel components of the magnetic fields at the boundaries being about three and the density ratio being 10 to 30 . The bipolar electric field and quadrupolar magnetic field of symmetric reconnection are not found in either the simulations or the experimental data because the Hall term in the generalized Ohm's Law (the leading term on its right side on the ion scale) is a factor up to 100 smaller on the magnetosheath side of the current layer than on the magnetospheric side. Average plasma densities and fields at six THEMIS magnetopause crossings agree with open simulations provided that the experimental data is in the joint variance, not minimum variance, frame. This is because the magnetic field component normal to the assumed planar current sheet is not constant for asymmetric reconnection while it is forced to be approximately constant by the mathematics of minimum variance analyses. A north-south asymmetry of the fields and flows in asymmetric reconnection is found in both the simulations and the space measurements. The simulations and the satellite data both have regions the order of the electron skin depth in size that contain significant electric fields. In such structures, parallel electric fields exist in the simulation throughout the current layer. Such parallel fields are similar to those reported earlier from Polar satellite data.

Citation: Mozer, F. S., P. L. Pritchett, J. Bonnell, D. Sundkvist, and M. T. Chang (2008), Observations and simulations of asymmetric magnetic field reconnection, J. Geophys. Res., 113, A00C03, doi:10.1029/2008JA013535.

\section{Introduction}

[2] Symmetric magnetic field reconnection is defined as reconnection when both the magnitude of the reconnecting magnetic field (the $\mathrm{Z}$ component in this paper) and the plasma density are the same on both sides of the current sheet. Much of the reconnection literature involves symmetric reconnection, which has been observed in simulations [Birn et al., 2001, and references therein], space [Mozer et al., 2002; Wygant et al., 2005], and the laboratory [Ren et al., 2005]. Characteristics of symmetric reconnection are a quadrupolar out-of-plane (Y direction) magnetic field component and bipolar normal (X component) electric field, as illustrated in the Polar satellite example of Figure 1 [Mozer et al., 2002]. Note that the plasma densities on the two sides of the current sheet in Figure 1a are the same within a factor of two and the magnitudes of $B_{Z}$ in the asymptotic regions of Figure 1e are the same. The quadrupolar magnetic field is manifested in Figure 1d as the positive and negative excursions in the out-of-plane com-

\footnotetext{
${ }^{1}$ Space Sciences Laboratory, University of California, Berkeley, California, USA.

${ }^{2}$ Department of Physics and Astronomy, University of California, Los Angeles, California, USA.

Copyright 2008 by the American Geophysical Union. 0148-0227/08/2008JA013535\$09.00
}

ponent, $\mathrm{B}_{\mathrm{Y}}$. The bipolar electric field in Figure 1f points toward the center of the current sheet from each side.

[3] In the terrestrial magnetosphere, symmetric reconnection is more frequently observed in the tail (because of the symmetry above and below the midplane) than at the dayside magnetopause where the plasma density and the reconnecting magnetic field may each vary by more than an order of magnitude across the current layer. Thus, dayside terrestrial reconnection is generally characterized by asymmetric conditions that are observed to lead to very different field and flow geometries than those associated with symmetric reconnection and illustrated in Figure 1. Examples of these different geometries observed in experiment [Mozer et al., 2008] and simulations [Pritchett, 2008] as well as recent theories of asymmetric reconnection [Borovsky and Hesse, 2007; Cassak and Shay, 2007] have been published. It is the purpose of this paper to compare simulations and space observations to elucidate some properties of asymmetric magnetic field reconnection.

[4] The physics behind the differences between symmetric and asymmetric reconnection is contained in the Hall term of the Generalized Ohm's Law which is

$$
\mathbf{E}+\mathbf{U}_{\mathrm{I}} \times \mathbf{B}=\mathbf{j} \times \mathbf{B} / \mathrm{en}-\nabla \cdot \mathbf{P}_{\mathrm{e}} / \mathrm{en}+\left(\mathrm{m}_{\mathrm{e}} / \mathrm{ne}^{2}\right) \delta \mathbf{j} / \delta \mathrm{t}+\eta \mathbf{j}
$$

where $\mathbf{E}$ and $\mathbf{B}$ are the electric and magnetic fields, $\mathbf{U}_{\mathrm{I}}$ is the ion fluid flow, $\mathbf{j}$ is the current density, $\mathrm{n}$ is the plasma density, $\eta$ is the resistivity associated with ion-electron 


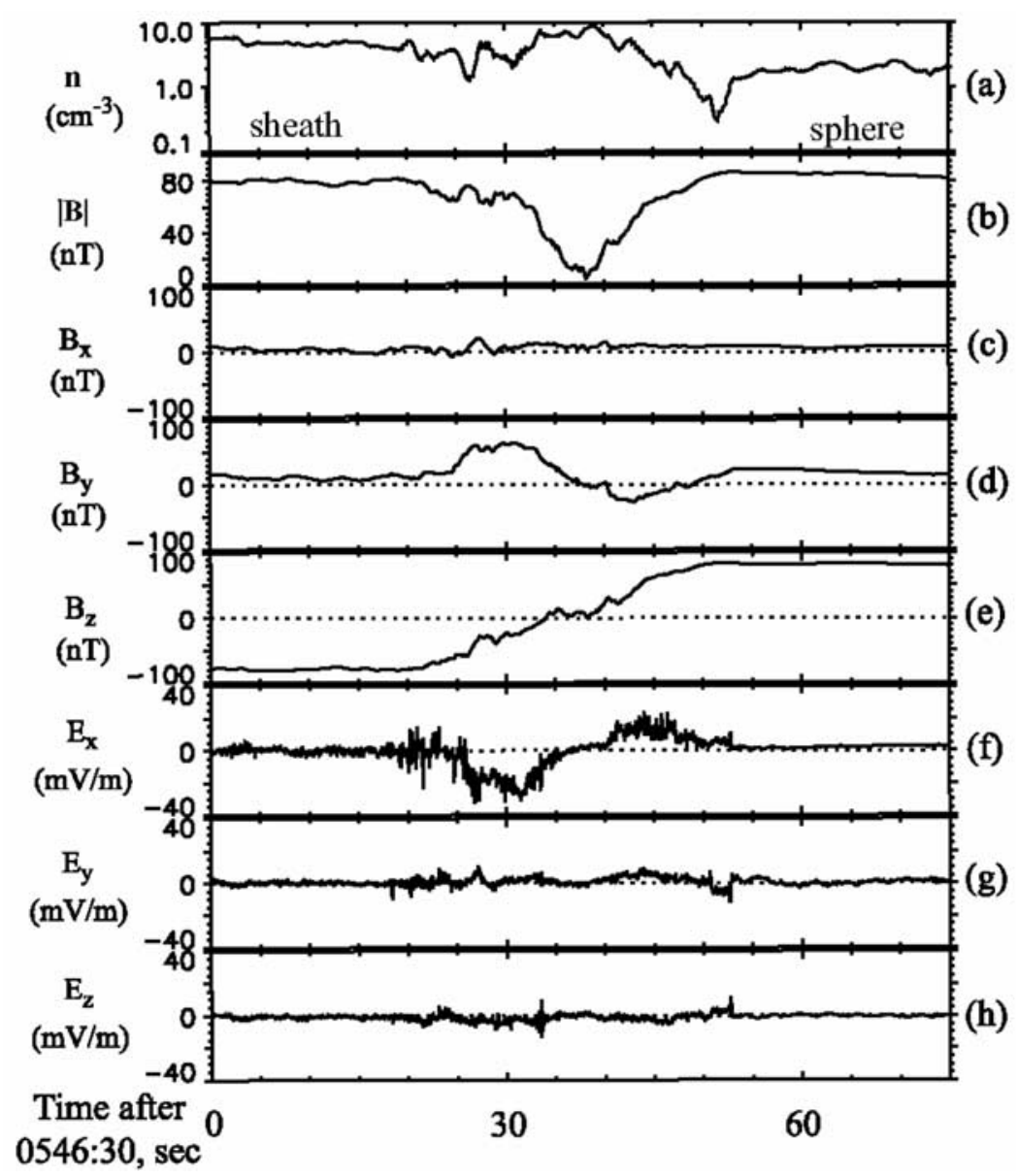

Figure 1. Electric and magnetic fields in symmetric reconnection.

interactions, and $\nabla \cdot \mathbf{P}_{\mathrm{e}}$ is the divergence of the electron pressure tensor. The first term on the right side of this equation is the Hall term. It will be shown that the major differences between symmetric and asymmetric reconnection stem from differences in the Hall term on the two sides of the current sheet for asymmetric reconnection due to different densities and magnetic fields on the two sides [Mozer et al., 2008].

\section{Simulation and Space Data Sources}

[5] The PIC simulation model used in this paper is the same as that in the work of Pritchett [2008] although that model utilized closed boundary conditions and the current results are for open boundary conditions, as discussed below. The asymmetric reconnection configuration is modeled by a hydromagnetic equilibrium in which the magnetic field profile is

$$
\mathrm{B}_{0 \mathrm{z}}(\mathrm{x})=\mathrm{B}_{0}[\tanh (\mathrm{x} / \lambda)+\mathrm{R}]
$$

and the density variation is

$$
\mathrm{n}(\mathrm{x})=\mathrm{n}_{0}\left[1-\alpha \tanh (\mathrm{x} / \lambda)-\alpha \tanh ^{2}(\mathrm{x} / \lambda)\right]
$$

The pressure balance constraint is satisfied if $\mathrm{R}=1 / 2$ (giving a field variation from $-\mathrm{B}_{0} / 2$ on the magnetosheath side to $3 \mathrm{~B}_{0} / 2$ on the magnetosphere side $)$ and $\alpha \mathrm{n}_{0}\left(\mathrm{~T}_{\mathrm{i}}+\mathrm{T}_{\mathrm{e}}\right)=$ $\mathrm{B}_{0}^{2} / 8 \pi$, where the temperatures are assumed to be uniform. (The effect of nonuniform temperatures is unknown.) For all the simulations discussed in this paper, $\mathrm{T}_{\mathrm{i}}=2 \mathrm{~T}_{\mathrm{e}}$ and $\alpha=$ 0.45 . The density variation across the current layer is then from $\mathrm{n}_{0}$ on the magnetosheath side to $\mathrm{n}_{0} / 10$ on the magnetosphere side. For comparison with the experimental data in this paper $\mathrm{B}_{0}$ is set to $36 \mathrm{nT}, \mathrm{n}_{0}$ is set to 25 particles/ $\mathrm{cm}^{3}$ and the normalized electric field of the simulation is multiplied by $v_{0}$ times $B_{0}(5.6 \mathrm{mV} / \mathrm{m})$ where $v_{0}$ is the Alfven speed determined from $B_{0}$ and $n_{0}$. The speed of light in the simulation is given by $\mathrm{c} / \mathrm{v}_{0}=20$. The ion to electron mass ratio is $\mathrm{m}_{\mathrm{i}} / \mathrm{m}_{\mathrm{e}}=200$, the scale length $\lambda=0.5 \mathrm{c} / \omega_{\text {pi }}$, the ion inertia length $\mathrm{d}_{\mathrm{i}}=\mathrm{c} / \omega_{\mathrm{pi}}=40 \Delta$ based on the density $\mathrm{n}_{0}$ ( $\Delta$ is the grid spacing), and the magnetosheath ion beta value based on the field, $\mathrm{B}_{0} / 2$, is $\beta_{\mathrm{i} \text {,sh }}=5.9$. In addition to the field $\mathrm{B}_{0 Z}(\mathrm{x})$, a uniform guide field $\mathrm{B}_{0 Y}$ equal to the magnetosheath field $\mathrm{B}_{0} / 2$ (directed dawnward) is present initially. This gives a shear angle of $117^{\circ}$ across the current layer. The simulation domain size is $25.6 \mathrm{~d}_{\mathrm{i}} \times 25.6 \mathrm{~d}_{\mathrm{i}}$.

[6] In simulation plots, distances are normalized to $d_{i}$ (which is between 40 and $200 \mathrm{~km}$ in the space data) and times to the inverse ion cyclotron time $\Omega_{\mathrm{i} 0}^{-1}=\mathrm{m}_{\mathrm{i}} \mathrm{c} / \mathrm{eB}_{0}$ (which is about $290 \mathrm{~ms}$ for $\mathrm{B}_{0}=36 \mathrm{nT}$ ). The simulation data are presented in a current sheet normal coordinate system where positive $\mathrm{X}$ points from the Earth to the Sun, $\mathrm{Y}$ is duskward, and $\mathrm{Z}$ points northward. 


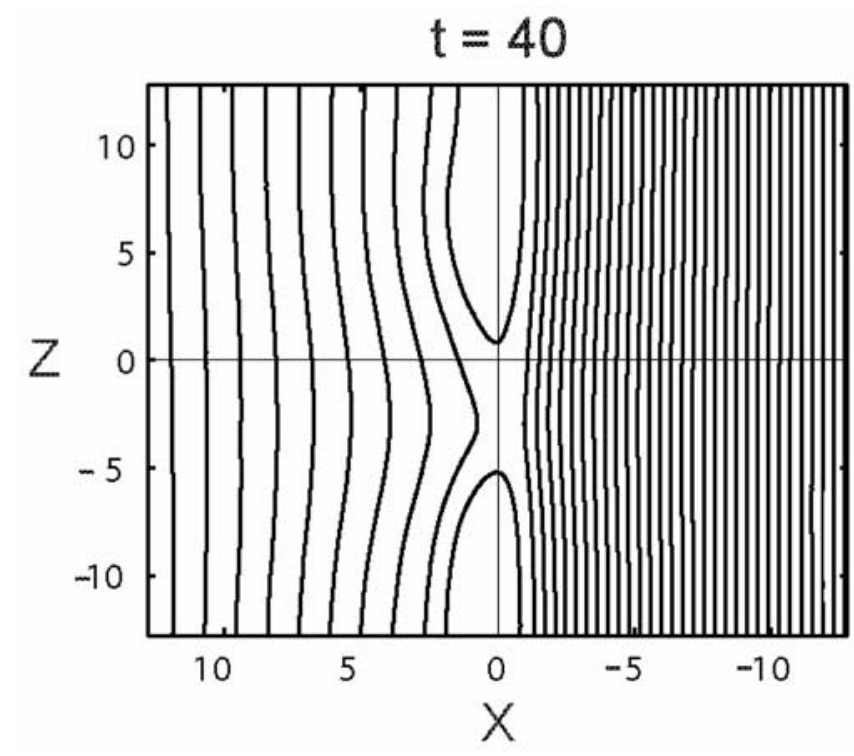

Figure 2. Magnetic field lines in a two-dimensional plane for asymmetric reconnection.

[7] The simulations are initialized with a flux perturbation $\delta \psi$ to locate the $\mathrm{X}$ line in the center of the system; the maximum strength of the perturbation is $\delta \psi_{0}=0.05 \mathrm{~B}_{0} \mathrm{c} / \omega_{\mathrm{pi}}$. No external convection electric field is applied to the system, so the simulations are undriven [Pritchett, 2008]. This undriven simulation may be appropriate for comparison with the experimental data collected at a subsolar distance of $12 \mathrm{R}_{\mathrm{E}}$ because this location is beyond the average position of the magnetopause, so the incident flow may be less driven than if the magnetopause was closer to the Earth. The $\mathrm{X}$ boundaries are assumed to be perfect conductors where particles are reflected back into the system. The $\mathrm{Z}$ boundaries are open to particles and magnetic flux: particles crossing such boundaries are removed from the system, and new particles are injected at a constant rate based upon a thermal Maxwellian distributions with $T_{I}$ and $T_{e}$ fixed at their initial values. In order to allow magnetic flux to escape, the perturbed field $\delta \mathrm{B}_{Z}=0$ at the $\mathrm{Z}$ boundaries, corresponding to a zero-slope condition on the vector potential $\delta \mathrm{A}_{Y}$. Likewise, $\delta \mathrm{B}_{\mathrm{Y}}$ is taken to vanish at these boundaries.

[8] The experimental data comes primarily from two subsolar magnetopause crossings by each of three THEMIS spacecraft, which have been described previously [Mozer et al., 2008]. Also included is a magnetopause crossing by Polar [Mozer et al., 2002].

\section{Features of Asymmetric Reconnection}

[9] Figure 2 presents a simulation overview of the asymmetric reconnection magnetic field geometry in a twodimensional plane. In this and all simulation figures, the magnetosheath is to the left and the measurement snapshot is made at $t=40$. During this 40 time units from the start of the simulation, the $X$ line moved from the origin to $\mathrm{Z} \sim-3.2 \mathrm{~d}_{\mathrm{i}}$ as a result of the $\mathrm{X}$ line drift in the direction of the electron diamagnetic drift [Swisdak et al., 2003; Pritchett, 2008]. As a result of the asymmetry in field strengths on the two sides, the magnetic field lines near the magnetosheath are more curved than those near the magnetosphere, which suggests that $\mathrm{B}_{\mathrm{X}}$ on the magnetosheath side may be different from $\mathrm{B}_{\mathrm{X}}$ on the magnetospheric side of the current sheet, an important feature unique to asymmetric reconnection and discussed in detail below. Figure 3 (left) presents simulation plots of plasma density, magnetic field, and electric field as functions of $\mathrm{X}$ at $\mathrm{Z}=-6$. Figure 3 (right) presents the average fields and density found in a superposed epoch analysis of the spin-period-averaged fields measured during six THEMIS magnetopause crossings [Mozer et al., 2008]. (Averages of the space data, rather than the higher time resolution values that are discussed later, are used in order to average out the variations from crossing to crossing.) As suggested by Figure 2, $B_{X}$ in Figure $3 b$ (left) varies with $\mathrm{X}$ and is larger near the magnetosheath. That $\mathrm{B}_{\mathrm{X}}$ varies with $\mathrm{X}$ is emphasized in Figure $4 a$, which shows that $B_{X}$ is positive south of the $\mathrm{X}$ line and negative north of the $\mathrm{X}$ line on the magnetosheath side of the current sheet. This variation of $\mathrm{B}_{\mathrm{X}}$ with $\mathrm{X}$ is the first result of asymmetric reconnection that differs from that found for symmetric reconnection where $B_{X}$ is not a function of $X$. Note that in Figure 4 and all later simulation figures, the scales of the quantities in each of the columns differ from each other.

[10] $\mathrm{B}_{\mathrm{X}}$ measured in space and given by Figure $3 \mathrm{~b}$ (right), shows the same dependence on $\mathrm{X}$ as does the simulation. This is because the space data is presented in the joint variance coordinate system [Mozer and Retino, 2007] in which the normal direction (the $\mathrm{X}$ direction) is given by the maximum variance direction of the electric field. This maximum variance coordinate system is then converted to joint variance coordinates by rotation about the $x$ axis until its $\mathrm{Z}$ direction lines up closest to the $\mathrm{Z}$ direction found from a minimum variance analysis. The space data is consistent with the simulation for crossings south of the $\mathrm{X}$ line. From the fact that the reconnection outflow was southward for all six crossings, this sign of $\mathrm{B}_{\mathrm{X}}$ is as expected from the simulation.

[11] It is noted that in the minimum variance coordinate system, $B_{X}$ is essentially constant (not shown), as expected from the mathematical, not physical, properties of the minimum variance analysis. Because this constancy is inconsistent with the asymmetric simulation, a minimum variance analysis of the magnetic field provides an incorrect estimate of the direction normal to the current sheet. This point is emphasized by the facts that the averages of $\mathrm{B}_{\mathrm{X}}$ over each of the six crossings has the expected positive value for the joint variance analysis and trajectories south of the $\mathrm{X}$ line while the minimum variance analysis produces the correct sign of $\left\langle\mathrm{B}_{\mathrm{X}}\right\rangle$ for only half of the six crossings.

[12] The experimental and simulation plasma densities of Figure $3 \mathrm{a}$ are in agreement in that they both show a relative minimum of plasma density near the high-density magnetosheath. This minimum is also exhibited in Figure 5, which gives the plasma density over the reconnection region. In agreement with experimental data, the plasma density on the magnetospheric side of the density minimum is greater than on the magnetosheath side. The density dip occurs at the magnetosheath separatrix. It appears to result from the electron parallel flow along the separatrix in an attempt to reduce the density difference between the two sides of the 

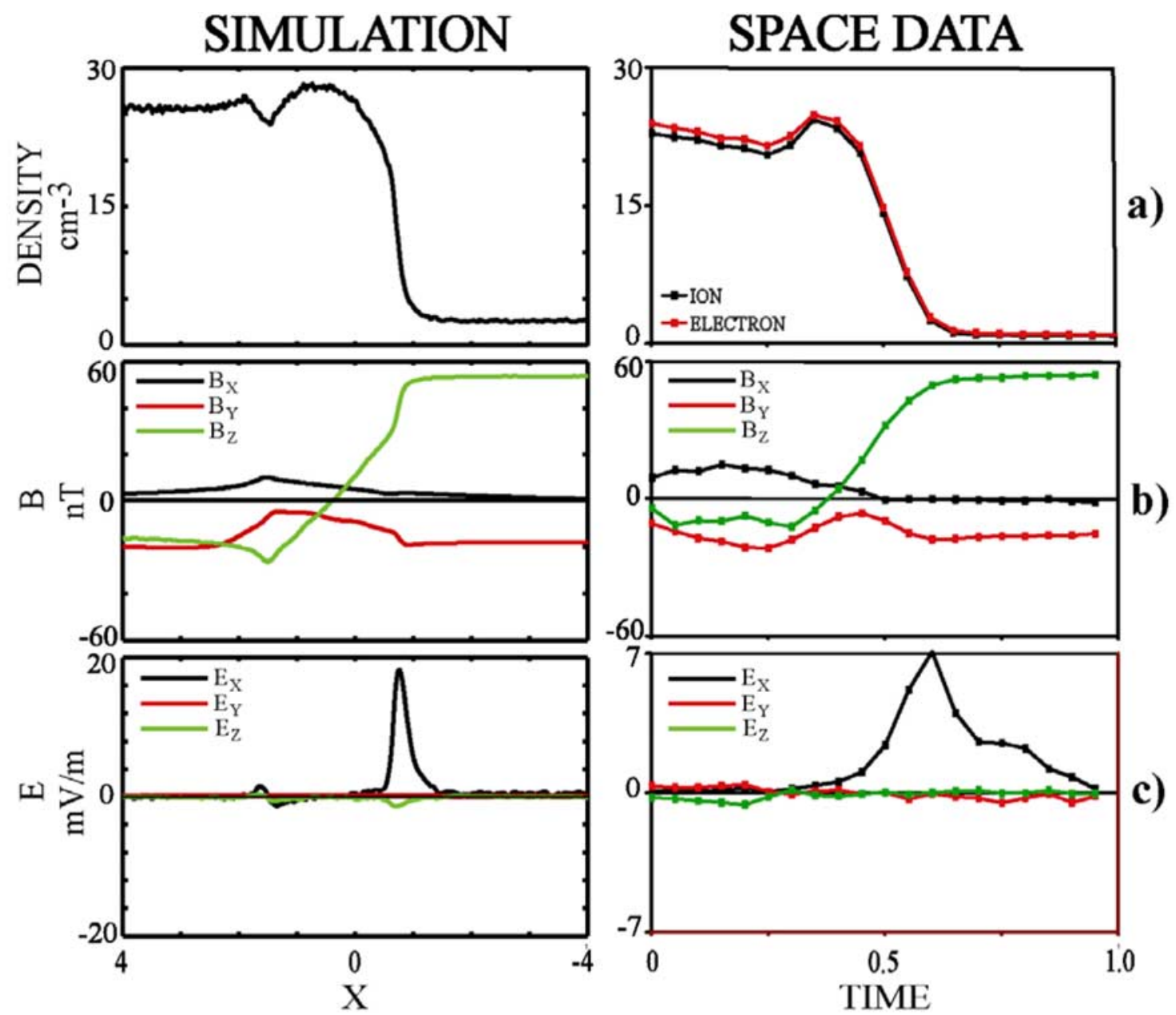

Figure 3. Comparison of plasma density and fields between a simulation and subsolar observations of asymmetric reconnection on the THEMIS satellite.

current layer together with an ion flow in $\mathrm{x}$ into the interior of the island

[13] $B_{Y}$ values as a function of $X$ are plotted in Figure $3 b$. They show agreement between the simulation and measurement in that $\mathrm{B}_{\mathrm{Y}}$ has a single dip toward zero from its average negative value that arises from the negative guide field. It is noted that contrary to the situation of a quadrupolar out-of-plane magnetic field in the symmetric reconnection case, there is only a single dip in this component of the magnetic field. This point is emphasized in Figure 4b, which gives $\mathrm{B}_{\mathrm{Y}}$ everywhere in the reconnection simulation. The blue color in Figure 4 arises from the average guide magnetic field. The average experimental data shows a single dip toward zero while the simulation shows two such dips in the southern hemisphere. The variation of $B_{Y}$ is opposite in the northern hemisphere, which causes the magnitude of $\mathrm{B}_{\mathrm{Y}}$ to increase in the northern hemisphere while it decreases in the southern hemisphere. This hemispheric difference, along with the north-south asymmetry of $\mathrm{B}_{\mathrm{X}}$ in Figure $4 \mathrm{a}$, results in the $\mathbf{E} \times \mathbf{B} / \mathrm{B}^{2}$ flows, the current $\mathbf{j}$, the Hall term and $\mathbf{j} \cdot \mathbf{E}$ having north-south asymmetries, which is another significant difference between symmetric and asymmetric reconnection. The north-south asymmetry is also evident: (1) in Figure 6a which shows an $(\mathbf{E} \times \mathbf{B}$ / $\left.\mathrm{B}^{2}\right)_{\mathrm{X}}$ inflow of field lines and Poynting flux across both separatricies in the northern hemisphere and an outflow at both separatricies in the southern hemisphere, (2) in Figure 6c which shows more $\mathrm{Z}$ outflow through the current sheet in the northern hemisphere than in the southern hemisphere, (3) in Figure 7 a which shows a smaller value of the magnetosheath $E_{X}$ in the southern hemisphere, (4) in Figure 7b, which shows a more positive $\mathrm{E}_{\mathrm{Y}}$ in the southern hemisphere, (5) in Figure $7 c$, which shows opposite signs of $E_{Z}$ at the separatricies in the two hemispheres, (6) in Figure 10 (left) that shows different structures of the parallel electric field in the two hemispheres.

[14] The north-south asymmetry would be inverted if the guide magnetic field was positive instead of negative, as it is in this data. If there was no guide magnetic field, the asymmetry would presumably disappear.

[15] The simulation and measured electric field components may be compared by referring to Figure 3c. In both cases there is a single peak in $\mathrm{E}_{\mathrm{X}}$, which is an electric field pointing toward the magnetosheath on the magnetospheric 


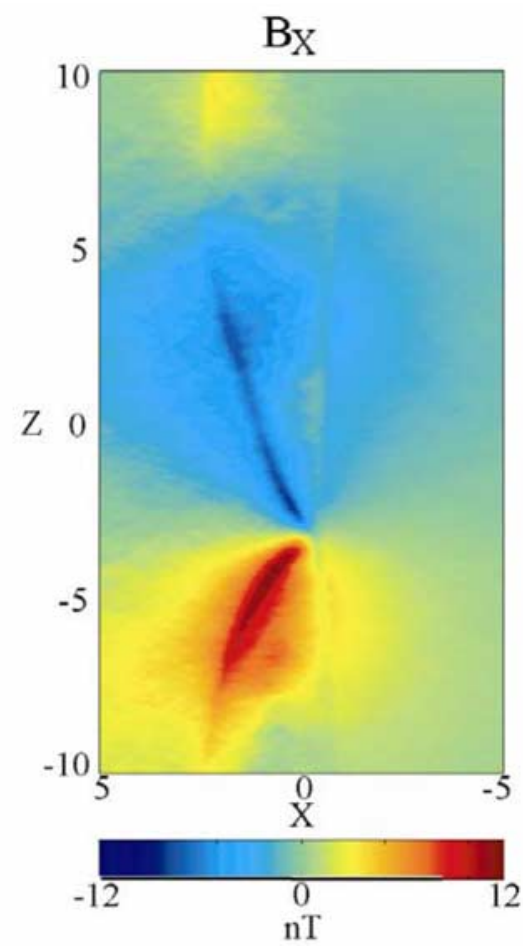

A
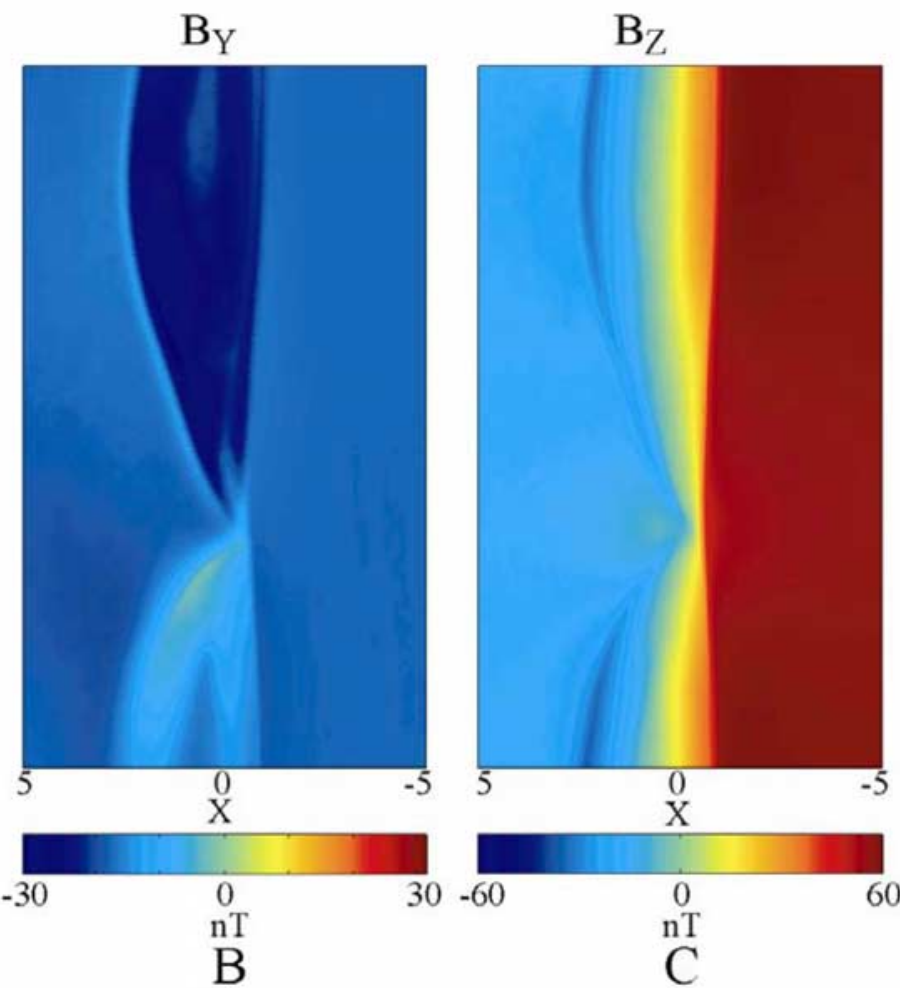

Figure 4. Magnetic field components in a two-dimensional plane, from a computer simulation of asymmetric reconnection. Note that the color scales for the field magnitudes differ for each component.

side of the reconnection layer. The existence of this peak and the absence of the bipolar component on the magnetosheath side (that is found in symmetric reconnection) is explained by the fact that the Hall term in the Generalized Ohm's Law, which is the leading term on the right side of equation (1) on the ion scale, differs by two orders of magnitude on the two sides [Mozer et al., 2008] while it is similar on the two sides for symmetric reconnection. This difference in the electric fields is associated with different particle motions and currents in the two reconnection cases, which is consistent with the quadrupolar magnetic field not being seen in asymmetric reconnection.

[16] The measured $\mathrm{Y}$ and $\mathrm{Z}$ components of the electric field are fractions of a $\mathrm{mV} / \mathrm{m}$, as shown in Figure $3 \mathrm{~b}$ (right) This is as expected from the simulation data in Figure $3 \mathrm{c}$ (left). However, the measured fields are comparable to experimental errors so no conclusions on the tangential components of the space electric field are possible.

[17] A plot of $E_{X}$ in a confined portion of the reconnection region is presented in Figure $7 \mathrm{a}$. The weak signatures in the southern magnetosheath side of the current sheet (which is where the satellite data was collected) are not observed in the average satellite data, perhaps because of signal-to-noise considerations and because these small signals may be averaged out in the summary plots of Figure 3 (right).

[18] The simulation $E_{X}$ has a resolution in $X$ that is less than that of the average data of Figure $3 c$ (right), as can be seen because the experimental $E_{X}$ has a duration comparable to that of the change of $B_{Z}$ while the simulation $E_{X}$ in Figure $3 \mathrm{c}$ (left) has a shorter duration. This is due, at least in part, to the averaging of the satellite data which also causes

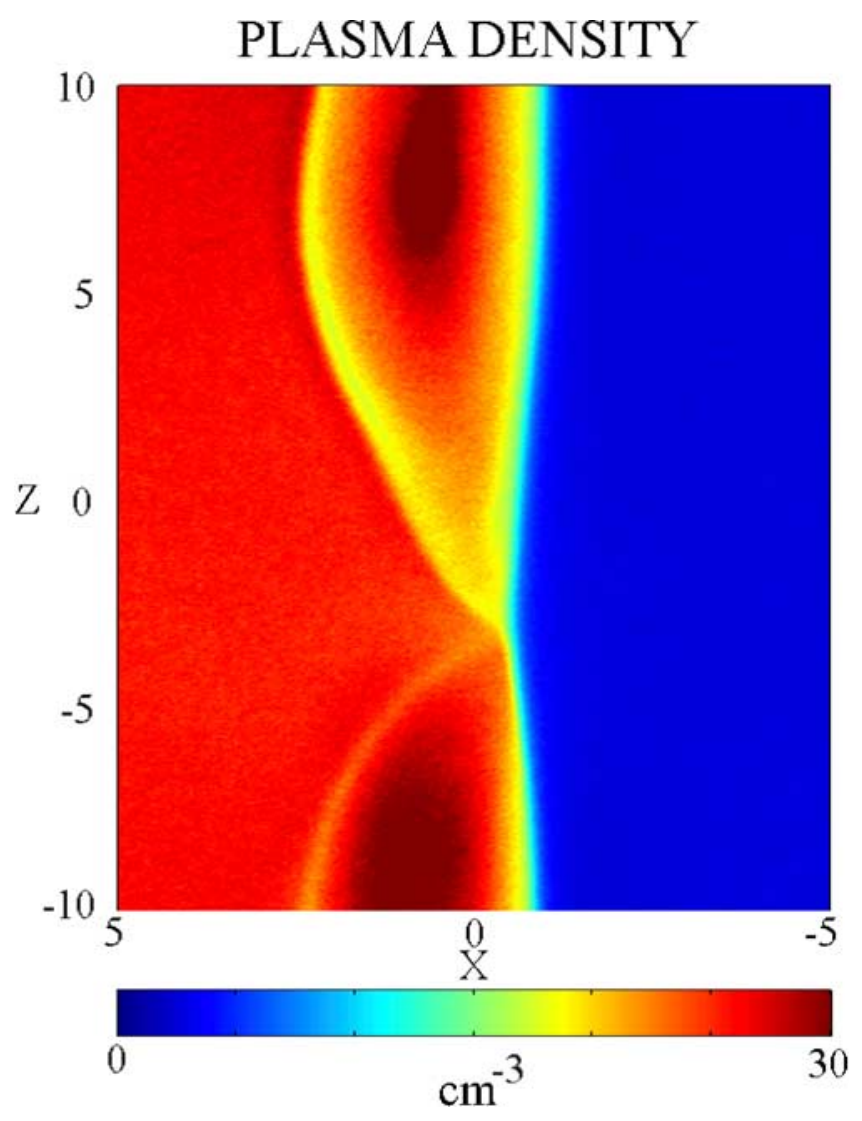

Figure 5. Plasma density in a two-dimensional plane from a computer simulation of asymmetric reconnection. 

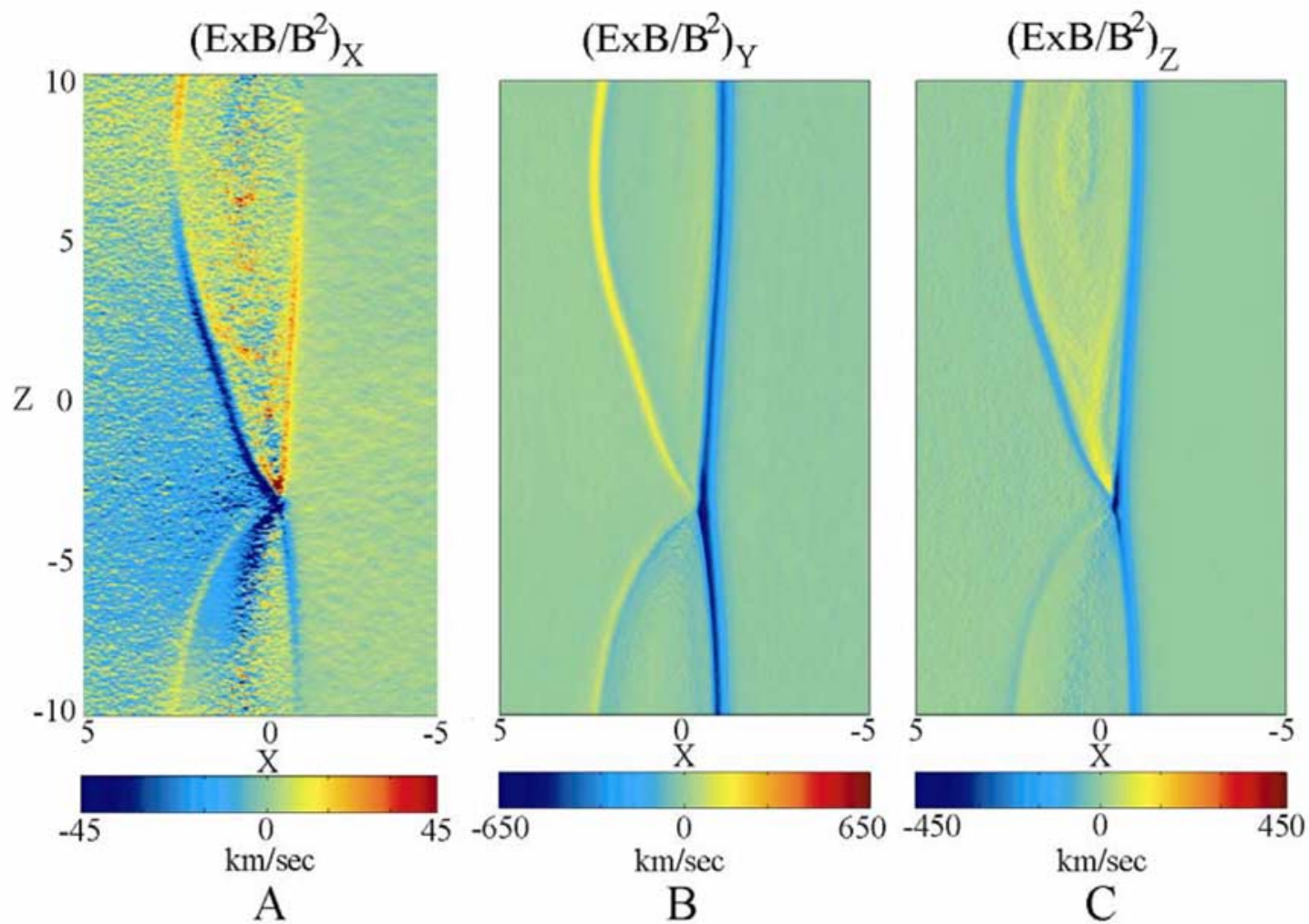

Figure 6. Components of $\mathbf{E} \times \mathbf{B} / \mathrm{B}^{2}$ in a two-dimensional plane, from a computer simulation of asymmetric reconnection. Note that the color scales for the field magnitudes differ for each component.
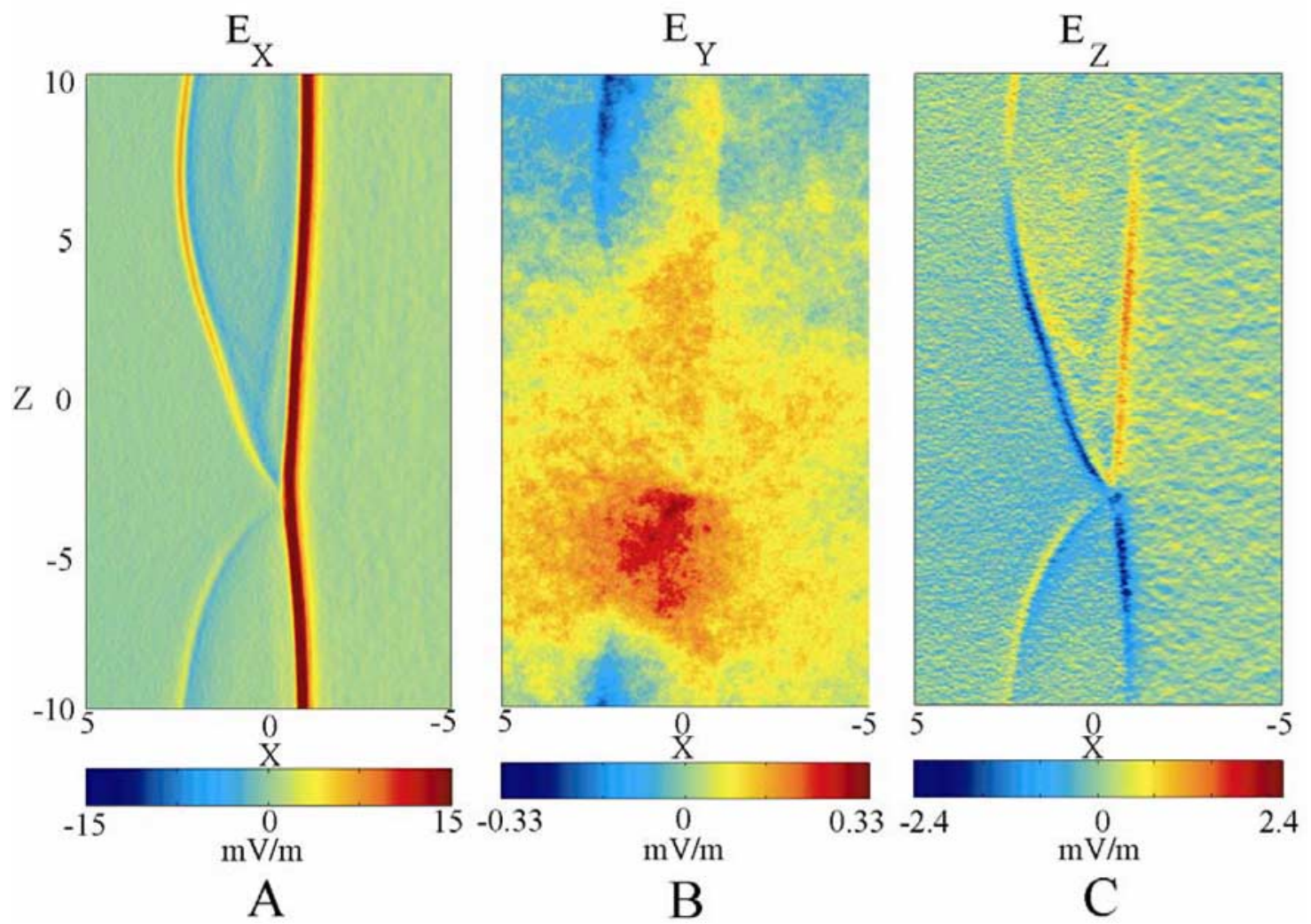

Figure 7. Electric field components in a two-dimensional plane, from a computer simulation of asymmetric reconnection. Note that the color scales for the field magnitudes differ for each component. 


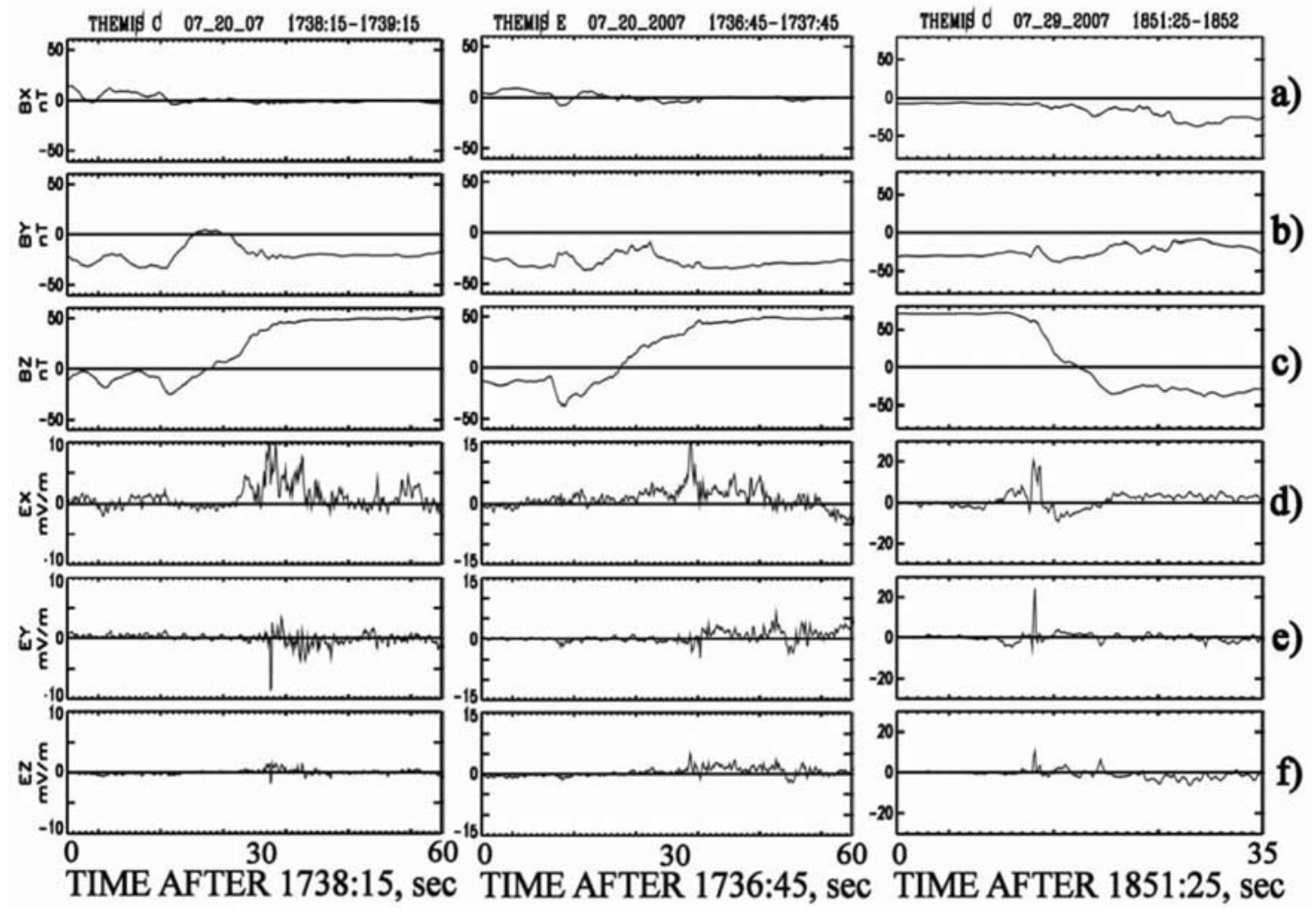

Figure 8. Three examples of THEMIS satellite crossings of reconnecting current sheets.

the peak amplitude of the space data to be smaller than that of the simulation. The $\sim 1 \mathrm{kV}$ potential drop associated with $\mathrm{E}_{\mathrm{X}}$ at the magnetospheric separatrix has the proper direction and magnitude to stop incoming magnetosheath ions and to divert them into the outflow channel.

[19] The structure of $E_{X}$ is further investigated by plotting high time resolution (eight points/second) examples of the fields in three different satellite crossings in Figure 8. Figures 8 (left and middle) include data that were averaged into the plots of Figure 3, while, unlike the other satellite data, Figure 8 (right) was collected during a pass from the magnetosphere to the magnetosheath. $\mathrm{E}_{\mathrm{X}}$ of Figure 8d (left) has a duration comparable to that of the current sheet (determined from the change of $\mathrm{B}_{\mathrm{Z}}$ ), while in Figure $8 \mathrm{~d}$ (middle and right), the large $\mathrm{E}_{\mathrm{X}}$ has a much shorter duration than that of the current sheet. Thus, $E_{X}$ in these two events is consistent with the simulation in terms of the duration and amplitude of the $E_{X}$ signature, but there are variations of this parameter from crossing to crossing. It is also noted that $E_{X}$ of Figure 8 exists at the location of the maximum slope of $B_{Z}$, i.e., at the location of the maximum current density, as it does in the simulation of Figures $3 b$ and $3 c$ (left). This is because $\mathrm{E}_{\mathrm{X}}$ is determined by the Hall MHD term which depends on the current density [Mozer et al., 2008].

[20] $E_{Y}$ in a confined region of the simulation is presented in Figure $7 b$. Because the region of significant positive $E_{Y}$ grows over this time interval (not shown), the simulation is not in equilibrium. It is also unclear whether the satellite measurements were or were not made in a static situation.
Thus, $\mathrm{E}_{\mathrm{Y}}$ comparisons must be tentative. (In principle, this comment applies to all the field components. However, the only simulation component observed to change significantly between $t=30$ and $t=40$ is $E_{Y}$.) Even so, it is clear that the average $E_{Y}$ in both experiment and simulation is much less than $E_{X}$, as can be seen in Figure 3c. In Figure 8e (left and right) there are large values of $E_{Y}$ that are opposite in sign in the two cases. These examples illustrate the fact that it is difficult to generalize from averaged data or from measurements made with insufficient time resolution. It is also noted that negative values of $E_{Y}$ in Figures 7 and 8 are opposite in sign to that expected for the tangential electric field that drives plasma and electromagnetic energy into the current layer from both sides. In the simulation, the reverse $E_{Y}$ values propagate away from the $\mathrm{X}$ line region as a function of time, and thus, they may be partially an artifact of the $X$ line initialization. However, large negative values of $E_{Y}$ clearly exist in the space data, as is evidenced in Figure 8. Because, the peak value of $E_{Z}$ of Figure $7 \mathrm{c}$ is about seven times larger than that of $E_{Y}$, the main component of $(\mathbf{E} \times \mathbf{B}$ / $\left.\mathrm{B}^{2}\right)_{\mathrm{X}}$ near the separatricies is $\mathrm{E}_{\mathrm{Z}} \mathrm{B}_{\mathrm{Y}} / \mathrm{B}^{2}$ and not $\mathrm{E}_{\mathrm{Y}} \mathrm{B}_{\mathrm{Z}} / \mathrm{B}^{2}$ (as is sometimes assumed) because of the finite guide magnetic field. In the absence of the guide field, this component of flow and that through the current sheet might be very different.

[21] It is interesting to consider the timescales of electric field signatures in Figure 8. From the speeds of the crossings determined by fitting the Hall field to $(\mathbf{E}+\mathbf{v x B})$ [Mozer et al., 2008] traversal of an ion skin depth (c/ $\left.\omega_{\mathrm{pI}}\right)$ 


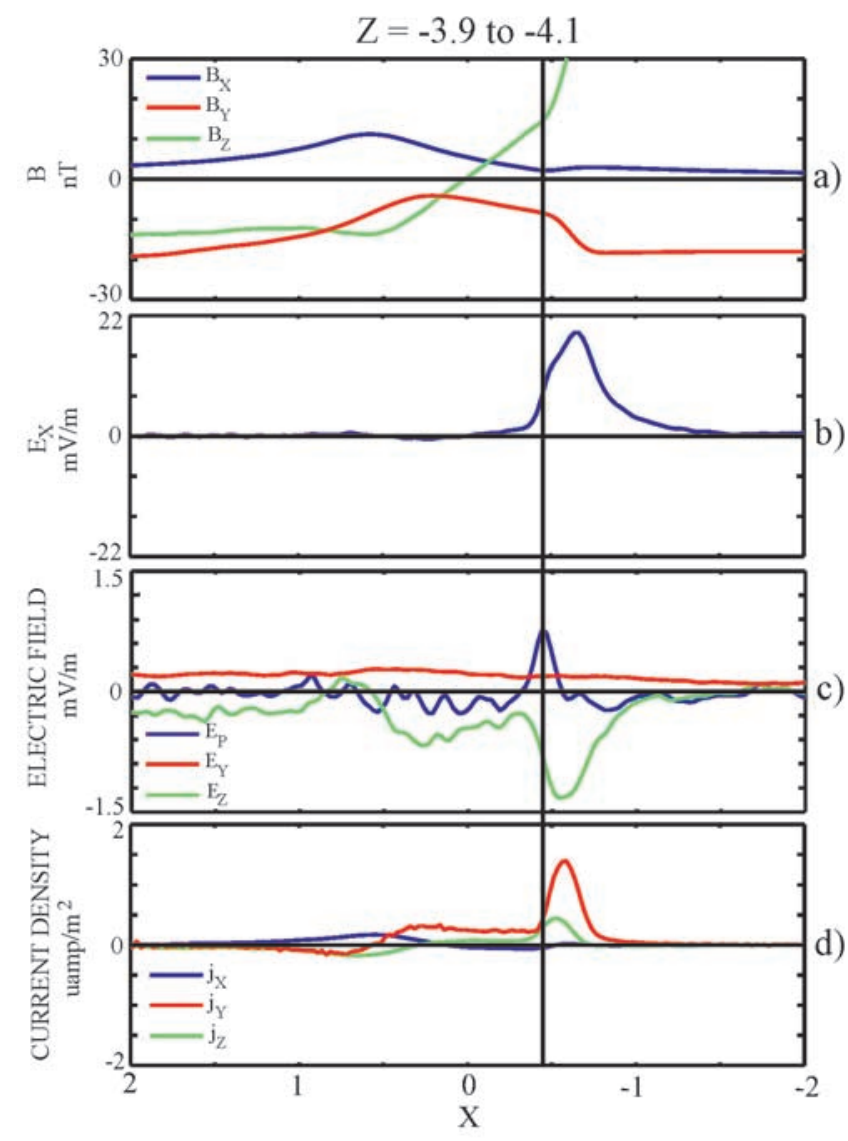

Figure 9. Simulation electric and magnetic fields and current density near an electron scale size region of a parallel electric field.

took about $10 \mathrm{~s}$. Thus, the current sheet widths in these examples are the order of a few ion skin depths. This is consistent with the simulation in that the width of the current sheet in Figures 2, 4, 5, 6, or 7 is a few ion skin depths, even relatively far from the $\mathrm{X}$ line.

[22] The electron skin depth $\left(\mathrm{c} / \omega_{\text {pe }}\right)$ was $2-3$ data points at the times of the $E_{Y}$ spikes in Figure 8 (left and right). These spikes had full widths at half maximum of about 1.5 data points. Thus, the large spikes in $\mathrm{E}_{\mathrm{Y}}$ existed on spatial scales less than or the order of the electron skin depth. If they also contained parallel electric fields, these would be candidate electron diffusion regions, meaning that they are of the electron scale size and the electrons are demagnetized. The THEMIS data does not allow an estimate of the parallel electric field so it cannot be determined whether these structures have such fields. However, the simulations contain significant parallel electric fields at such times, as is illustrated by the time domain data of Figure 9c, in which the parallel field is larger than $\mathrm{E}_{\mathrm{Y}}$ and comparable to $E_{Z}$. The full region plot of the parallel electric field is given in Figure 10 (left). The separatricies in Figure 10 (left) are those of the maxima of $E_{Z}$ in Figure $7 c$. Similar parallel electric fields exist through the entire current sheet in Figure 10. In agreement with this result, parallel electric fields on electron spatial scales have also been observed throughout the current sheet on the Polar satellite, which is capable of such measurements because it has a three component electric field experiment [Mozer et al., 2003; Mozer, 2005]. An example of one such measured parallel electric field is given in Figure 10e, 10f, and 10g (right) which give the electric field in magnetic field aligned coordinates with the $\mathrm{Z}$ component being parallel to $\mathbf{B}$. The parallel electric field of $9 \mathrm{mV} / \mathrm{m}$ existed in a region of size less than an electron skin depth. Its magnitude was about $20 \%$ of the $40 \mathrm{mV} / \mathrm{m}$ perpendicular electric field. The ratio of parallel to perpendicular electric fields in the simulation is about $5 \%$, as can be seen by comparing Figure $7 \mathrm{a}$ and Figure 10 (left). This difference in the relative sizes of the parallel electric fields in simulations and measurements may be explained by the facts that only the largest parallel fields are reported in the space data because of signal to noise considerations, and that the geocentric altitude of the space data in which parallel fields were observed was less than $10 \mathrm{R}_{\mathrm{E}}$, which may mean that a better comparison of parallel fields in space and simulation could be for driven rather than undriven simulations.

[23] The satellite measured average value of $E_{Z}$ in Figure $3 c$ (right) is close to zero. This differs from the simulation which shows a field along the separatricies that is about $5-10 \%$ of $E_{X}$, as may be seen by comparing Figures $7 \mathrm{a}$ and $7 \mathrm{c}$ or Figures $9 \mathrm{~b}$ and $9 \mathrm{c}$. This discrepancy may be partially due to averaging the data because nonzero values of $\mathrm{E}_{Z}$ are found in Figure $8 \mathrm{f}$. It is also partially due to the $E_{Z}$ field having a component parallel to $\mathrm{B}$ and, as discussed above, this component is not measured on THEMIS.

[24] In Figure 8a (left and middle) $\mathrm{B}_{\mathrm{X}}$ is more positive near the magnetosheath than near the magnetosphere while in Figure 8c (right) it is more negative (note that the magnetosheath is on the right side in Figure 8 (right)). These results are consistent with $\mathrm{B}_{\mathrm{X}}$ of Figure $4 \mathrm{a}$ because the ion outflow showed that the crossings of Figure 8 (left and middle) were south of the X-line while the Figure 8 (right) crossing was north of the $\mathrm{X}$ line. Also consistent with Figure $4 b$, the measured $B_{Y}$ tended toward zero in the current sheet in Figure 8 (left and middle) while it tended to be larger in magnitude in Figure 8 (right).

\section{Conclusions}

[25] Comparisons of asymmetric magnetic field reconnection between simulations and sub-solar magnetopause satellite data, averaged over several crossings, show excellent agreement in the spatial variations of the electric field components, the magnetic field components and the plasma density. In addition, parallel electric fields on the electron scale size are observed through the current sheet in both cases. Differences between symmetric and asymmetric reconnection include:

[26] 1. For asymmetric reconnection, there is a significant north-south asymmetry of the fields and flows while no such asymmetry exists for symmetric reconnection.

[27] 2. The quadrupolar out-of-plane magnetic field and bipolar in-plane electric field of symmetric reconnection are not found in the asymmetric case.

[28] 3. While a minimum variance analysis of satellite data may be appropriate for symmetric reconnection, it is not applicable to asymmetric reconnection because it gives 

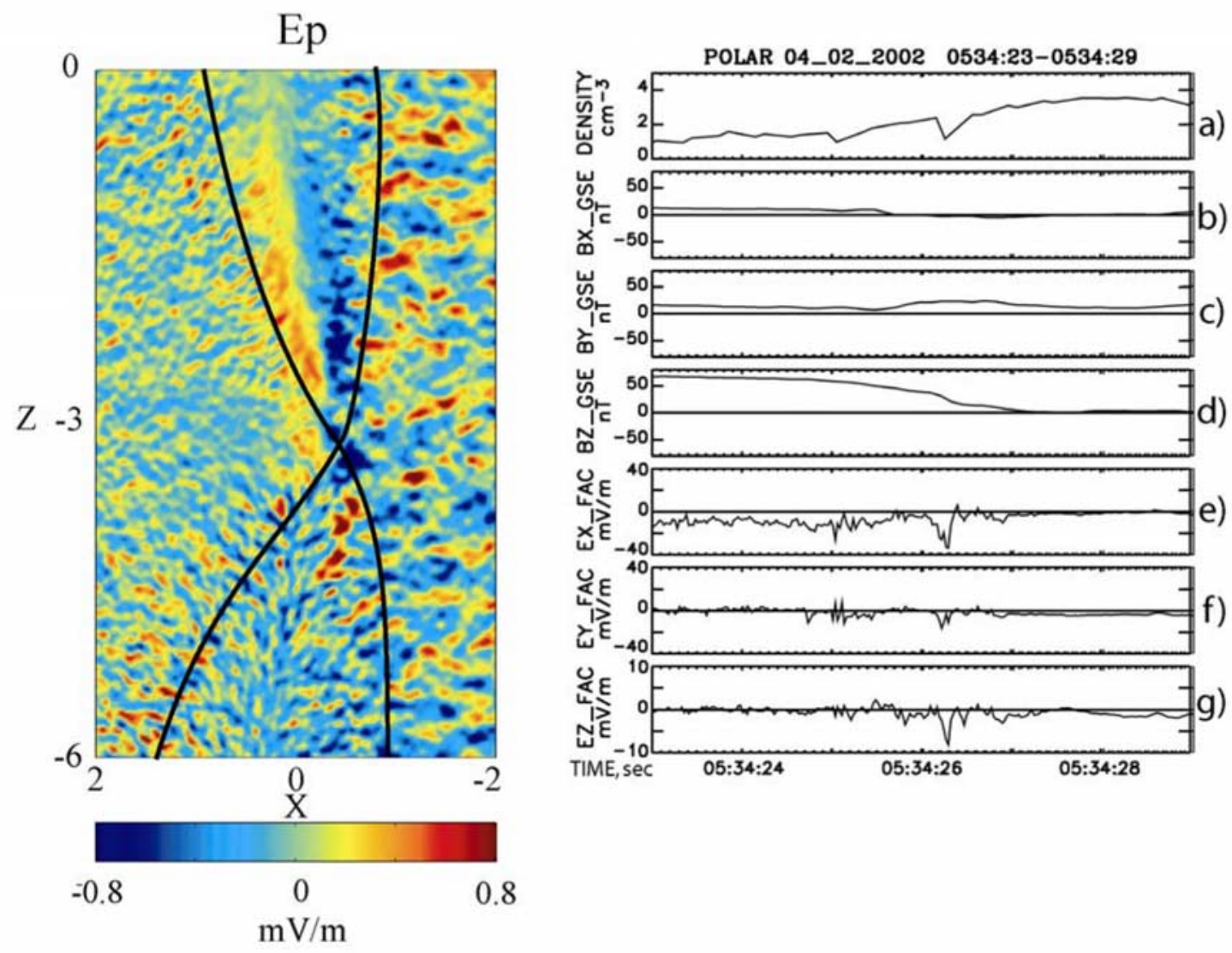

Figure 10. Simulation of significant parallel electric fields throughout a current sheet and a Polar satellite example of the direct observation of such a field. Note that the amplitude scale for the parallel electric field in Figure 10g (right) differs from those of the other electric field components.

an incorrect direction normal to the plane of the current sheet. Instead, a joint variance analysis of satellite data should be performed in the asymmetric case.

[29] Acknowledgments. This research was supported by NASA grants NNX08AM15G, NNG05GL27G, and NNG05GC72G and contract NAS5-02099-07/09. The particle simulations were performed using resources of the San Diego Supercomputer Center (supported by the National Science Foundation under cooperative agreement ACI-9619020) and the UCLA Dawson Cluster (funded by NSF grant PHY-0321345).

[30] Amitava Bhattacharjee thanks the reviewers for their assistance in evaluating this paper.

\section{References}

Birn, J., et al. (2001), Geospace environmental modeling (GEM) magnetic reconnection challenge, J. Geophys. Res., 106, 3715, doi:10.1029/ 1999JA900449.

Borovsky, J. E., and M. Hesse (2007), The reconnection of magnetic fields between plasmas with different densities: Scaling relations, Phys. Plasmas, 14, 102309, doi:10.1063/1.2772619.

Cassak, P. A., and M. A. Shay (2007), Scaling of asymmetric magnetic reconnection: General theory and collisional simulations, Phys. Plasmas, $14,10,211$.

Mozer, F. S. (2005), Criteria for and statistics of electron diffusion regions associated with sub-solar magnetic field reconnection, J. Geophys. Res., 110, A12222, doi:10.1029/2005JA011258.

Mozer, F. S., and A. Retinò (2007), Quantitative estimates of magnetic field reconnection properties from electric and magnetic field measurements, $J$. Geophys. Res., 112, A10206, doi:10.1029/2007JA012406.
Mozer, F. S., S. D. Bale, and T. D. Phan (2002), Evidence of diffusion regions at a sub-solar magnetopause crossing, Phys. Rev. Lett., 89, 015002, doi:10.1103/PhysRevLett.89.015002.

Mozer, F. S., S. D. Bale, T. D. Phan, and J. A. Osborne (2003), Observations of electron diffusion regions at the sub-solar magnetopause, Phys. Rev. Lett., 91, 245002, doi:10.1103/PhysRevLett.91.245002.

Mozer, F. S., V. Angelopoulos, J. Bonnell, K. H. Glassmeier, and J. P. Mcfadden (2008), THEMIS observations of modified Hall fields in asymmetric magnetic field reconnection, Geophys. Res. Lett., 35, L17S04, doi:10.1029/2007GL033033.

Pritchett, P. L. (2008), Collisionless magnetic reconnection in an asymmetric current sheet, J. Geophys. Res., 113, A06210, doi:10.1029/ 2007JA012930.

Ren, Y., et al. (2005), Experimental verification of the Hall effect during magnetic reconnection in a laboratory plasma, Phys. Rev. Lett., 95, 055003, doi:10.1103/PhysRevLett.95.055003.

Swisdak, M., B. N. Rogers, J. F. Drake, and M. A. Shay (2003), Diamagnetic suppression of component magnetic reconnection at the magnetopause, J. Geophys. Res., 108(A5), 1218, doi:10.1029/2002JA009726.

Wygant, J. R., et al. (2005), Cluster observations of an intense normal component of the electric field at a thin reconnecting current sheet in the tail and its role in the shock-like acceleration of the ion fluid into the separatrix region, J. Geophys. Res., 110, A09206, doi:10.1029/ 2004JA010708

J. Bonnell, M. T. Chang, F. S. Mozer, and D. Sundkvist, Space Sciences Laboratory, University of California, Berkeley, CA 94720, USA. (fmozer@ssl.berkeley.edu)

P. L. Pritchett, Department of Physics and Astronomy, University of California, Los Angeles, CA 90024-1547, USA. 